\title{
EL FIN DEL PARTIDO DE LA TRANSICIÓN. LA DIVISIÓN INTERNA COMO CAUSA DE LA DESAPARICIÓN DE UCD ${ }^{1}$
}

\author{
THE END OF THE PARTY OF THE TRANSITION. THE INTERNAL DIVISION
}

AS A CAUSE OF THE DISAPPEARANCE OF UCD

\author{
José Ignacio Villar Romero \\ Universidad de Córdoba \\ Córdoba University, Spain
}

ORCID: 0000-000I-6109-2090

jvillar@uco.es

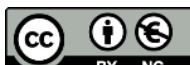

\begin{abstract}
Resumen
El auge y caída de Unión de Centro Democrático ha sido uno de los grandes interrogantes de la Historia Contemporánea de España. La desaparición del que se ha considerado como el partido de la Transición, se debió, entre otros factores, a la aguda división existente en la formación desde sus orígenes. Democristianos, socialdemócratas, liberales, azules, regionalistas, o sectores muy próximos a determinados barones, vivieron una lucha interna tan polémica como las que mantuvieron con los partidos de la oposición. En este artículo se analizará cómo las graves disputas internas se convirtieron en un factor precipitante del ocaso de UCD. Un proyecto político que lo había llegado a ser todo bajo el liderazgo de Adolfo Suárez, y que se desmembró por completo tan sólo cinco años después de su creación. En las elecciones generales de 1982 perdió la inmensa mayoria de su representación en el Congreso, pasando de 168 a II diputados. En 1983 se acordó su total disolución, si bien mantuvo su grupo parlamentario hasta 1986. El equilibrio y los juegos de poder entre las "familias", tanto en la estructura orgánica del partido, como en el grupo parlamentario, así como en el Gobierno; se convirtieron en un obstáculo insalvable para su consolidación, provocando así su descalabro.
\end{abstract}

Palabras clave: Transición; UCD; Adolfo Suárez; Democracia; Partidos políticos; Elecciones.

\section{Abstract}

The rise and fall of Unión de Centro Democrático has been one of the great questions of the Contemporary History of Spain. The disappearance of what has been considered the party of the Transition was due, among other factors, to the sharp division existing in the formation since its origins. Christian Democrats, social democrats, liberals, blues, regionalists, or sectors very close to certain barons, experienced an internal struggle as controversial as the ones they had with the opposition parties. This article will analyze how the serious internal disputes became a precipitating factor in the decline of the UCD. A political project that had become everything under the leadership of Adolfo Suárez, and which was completely dismembered only five years after its creation. In the 1982 general elections it lost the vast majority of its representation in Congress, going from 168 to II deputies. In 1983 its total dissolution was agreed, although it maintained its parliamentary group until 1986. The balance and the power games between the "families", both in the organic structure of the party and in the parliamentary group, as well as in the Government, became an insurmountable obstacle for its consolidation, thus causing its collapse.

Keywords: Transition; UCD; Adolfo Suárez; Democracy; Political Parties; Elections.

\footnotetext{
${ }^{1}$ Recibido/Received: |5/| |/202|
}

Aceptado/Accepted: 21/0I/2022 


\section{| Introducción. Unión de Centro Democrático: el partido de la Transición |}

\section{UCD: una "sopa de siglas"}

Antes de la convocatoria de elecciones prevista para el 15 de junio de 1977, el centro político, o al menos, las diferentes ideologías que se podían agrupar en torno al centro - ser considerablemente cercanas al mismo, se dividían entre múltiples y dispares formaciones políticas que con el nuevo sistema electoral basado en las circunscripciones provinciales y la Ley D'Hondt, iban a tener muy difícil obtener una representación lo suficientemente proporcional a sus votos. Hopkin los ha definido como "partidos-taxi" que no disponían de los recursos ni apoyos necesarios (Hopkin, 2000, p. 271). Este amplio espacio estaba ocupado por formaciones democristianas (Federación Popular Democrática, Izquierda Democrática, Izquierda Demócrata-Cristiana) liberales (Partido Demócrata, Partido Liberal, Partido Demócrata-Popular) o socialdemócratas (Federación de Partidos Socialdemócratas, Federación Socialdemócrata), entre otros.

Al mismo tiempo, desde $1972-73$ se había organizado un grupo de opositores moderados al franquismo, vinculados con la Asociación Católica Nacional de Propagandistas y que según Huneeus, habían apoyado al Rey Juan Carlos para la formación de una monarquía parlamentaria (Huneuus, 1985, p. 184). Su nombre, Tácito, proviene de la signatura con la que firmaban sus publicaciones en el diario $\mathrm{Ya}$, en el que escribían a nombre del historiador latino. Entre sus miembros más destacados se encontraban Alfonso Osorio, Landelino Lavilla, Marcelino Oreja o Calvo-Sotelo entre otros. $Y$ estos, junto con otros sectores tratados más adelante, actuarían como promotores para intentar agrupar al centro político en un solo ente común. Esta aspiración estaba marcada, en palabras de Alonso-Castrillo, por la llegada al poder de la izquierda en Portugal tras el fracaso de Caetano al intentar dirigir una transición política. Con la entrada de seis tácitos en el gobierno de Suárez en el año 1976 (Alfonso Osorio, Marcelino Oreja, Landelino Lavilla y Leopoldo Calvo-Sotelo) el grupo alcanzaba un punto de inflexión en el que su nuevo objetivo sería construir una formación política (Linares, 2013, p. 68).

El 10 de noviembre de 1976 se presentó en un acto el nuevo Partido Popular, proyecto político que había sido anunciado previamente en junio del mismo año y que trataba de configurarse como formación de centro independiente del gobierno de Suárez. Los mayores impulsores del PP eran Pío Cabanillas, José María de Areilza, José Pedro PérezLlorca y Emilio Attard. El PP parecía constituirse como la primera opción de gobierno centrista y democristiana capaz de llegar a un gobierno presidido hipotéticamente por Areilza, lo que para Huneuus era un auténtico desafío a Suárez.

EI PP, que representaba un espacio más abierto que exclusivamente el democristiano, aunaba en su seno tanto miembros del franquismo como opositores a él. Su enorme capacidad para atraer diferentes perfiles ideológicos iba a empujarlos a buscar la conformación de una coalición más amplia y con mayores posibilidades de ganar las 
elecciones. Así se configuró Centro Democrático, que integraba además de al PP, a los liberales del PDP liderados por Ignacio Camuñas-quien puso nombre a la coalición- y a la FPDP (Federación de Partidos Demócratas y Populares). Alfonso Osorio actuaba como agente de Suárez en esta estrategia de unificación del centro a la que pronto también se unirían el Partido Liberal de Enrique Larroque, el influyente Joaquín Garrigues Walker, o el Partido Popular Demócrata Cristiano de Fernando Álvarez de Miranda e Íñigo Cavero. También lo hicieron las diversas formaciones socialdemócratas, entre cuyos líderes destacaban Francisco Fernández Ordóñez, José Ramón Lasuén, o Rafael Arias-Salgado.

Solo hubo una ausencia notoria en la conformación de Centro Democrático. El ala democristiana que representaba Federación Demócrata Cristiana decidió mantenerse independiente y presentarse en solitario a las elecciones. FDC agrupaba a la Izquierda Democrática de Joaquín Ruiz Giménez -partidario de unirse a Centro Democrático- y a la Federación Popular Democrática de José María Gil-Robles, el histórico líder de la CEDA durante la Segunda República, que se negó a adherirse a la coalición centrista.

Huneuus sostiene que los movimientos anteriormente mencionados seguían dos objetivos: la alianza de los partidos de centro y derecha de la oposición, por un lado, y la autonomía política respecto del Gobierno por el otro (Huneuus, 1985, p. 155). Como se observa, ninguna de las pretensiones se había cumplido. Ruiz Giménez y Gil-Robles quedaban fuera, además de la concurrencia por separado de un pequeño sector de los liberales en las listas al Senado en las elecciones de 1977 encabezado por Joaquín Satrústegui. Y del mismo modo, era imposible mantener una total autonomía con respecto al Gobierno cuando dentro del mismo proyecto político se incluían miembros del ejecutivo de Suárez.

Si a estas contradicciones se suma el fracaso del primer y único acto de Centro Democrático en el mitin en Alicante del 2 de abril de 1977 en el que los oyentes apenas conocían a Fernández Ordóñez o Álvarez de Miranda, y al único al que reconocieronPío Cabanillas- algunos increparon por su pasado como ministro durante el franquismo, estaba claro que el centro necesitaba un líder fuerte y conocido por la población capaz de llegar a la Moncloa. Y el más indicado, como no, era el Presidente Suárez. No obstante, si bien Centro Democrático necesitaba al abulense, él también los necesitaba a ellos.

Corría la primavera de 1977 y Suárez había afianzado su liderazgo con decisiones como la legalización del PCE -aunque esto provocase la dimisión del almirante Pita da Veiga como Ministro de Marina-, o la visita a EEUU en mayo que reforzó su imagen exterior. Calvo-Sotelo se ocuparía de organizar las listas de la coalición de cara a los comicios, dejando el Ministerio de Obras Públicas para dedicarse a la que iba a ser una tarea harto compleja. Suárez y el gobierno contaban con los recursos de los que Centro Democrático no gozaba. 
Quince partidos constituyeron UCD en mayo de 1977, tan sólo un mes antes de las elecciones. Una auténtica "sopa de siglas" en la que además de democristianos, liberales, socialdemócratas o independientes; se integraron ahora a algunos regionalistas. En concreto, cinco de las quince formaciones no eran de carácter estatal. De este modo, había nacido una formación enormemente heterogénea, llena de diferentes familias ideológicas originarias de distintos partidos. La UCD según Gregorio Morán, era una “mezcla de retales-patchwork” (Morán, 2009, p. 160). Por su parte, Mario Caciagli habla de "demasiada" heterogeneidad el partido para recalcar que la construcción del partido acabó frustrada (Caciagli, 1989, p. 105). Y a pesar de aquellos complejos comienzos, ganaron las elecciones del 15 de junio con un 35\% del total de los votos (6.310.391) y 165 diputados en el Congreso, a tan sólo once de la mayoría absoluta. UCD pasaba de coalición a partido de gobierno. EI PSOE de Felipe González -con 118 diputados y un $30 \%$ de los votos- se erigía como primer partido de la oposición. Lejos quedaban el PCE de Carrillo con un $10 \%$ y la AP de Fraga con tan solo un $8 \%-20$ y 16 parlamentarios respectivamente-.

\section{La Legislatura Constituyente: primer Gobierno y primeras fricciones}

Con las elecciones ganadas y la Legislatura Constituyente comenzada, tanto el partido como el Gobierno debían redefinirse y alcanzar los equilibrios necesarios con el fin de evitar tensiones internas. Suárez formó un Ejecutivo en el que incluía a socialdemócratas, liberales, democristianos, miembros de Tácito y reformistas.

Es entonces cuando UCD se auto-organiza a través de un complejo proceso de distribución de cargos, poderes y responsabilidades. Se redactaron los Estatutos del partido, presentados por Salvador Sánchez-Terán, y en septiembre de 1977 el Consejo Político de UCD designó al primer Comité Ejecutivo. Suárez trasladó a la formación que el proyecto político debía basarse en el Gobierno, el partido y el grupo parlamentario lo que algunos han bautizado como "teoría del triciclo"-. Tanto Suárez como Fernando Abril Martorell ya tenían en mente la fusión total de UCD y la disolución de los partidos que lo formaban. Según Alonso-Castrillo, fue esta intención de Presidente y Vicepresidente junto con el uso del grupo parlamentario por parte del gobierno como instrumento propio, las razones que llevarían a Camuñas a dejar su cargo como Ministro (Alonso-Castrillo, 1996). Y efectivamente, en diciembre de 1977 se procedía a la disolución de las quince formaciones después de que estas lo aceptaran y el Comité Ejecutivo lo aprobara. Sólo Camuñas votó en contra de esta decisión. Álvarez de Miranda se abstuvo.

A comienzos de 1978 iba a producirse una nueva crisis de gobierno, esta vez mucho más profunda, y que tendría efectos colaterales internos en el partido. Ya poco antes habían causado baja Alfonso Osorio, que dejó el partido al posicionarse en contra de los Pactos de la Moncloa y la reforma fiscal, así como de las disoluciones de los partidos que integraban UCD. La marcha de Osorio arrastró más bajas, como la del senador González-Tarrio. 
Tras el éxito de los Pactos de la Moncloa, el mismo que los había impulsado, Enrique Fuentes Quintana (Vicepresidente $2^{\circ}$ y Ministro de Economía) trata de nacionalizar la Red de Alta Tensión. A esta iniciativa se niega rotundamente Alberto Oliart (Ministro de Industria), lo que provoca un enfrentamiento entre ambos. Suárez y Abril desechan la idea, y Fuentes Quintana presenta su renuncia el 23 de febrero de 1978. La nueva remodelación del gobierno iba a suponer un totum revolutum en las filas del partido. $Y$ es que según Jaúregui y Soriano, el mismo día se sabe que además varios ministros están “tocados" (Jaúregui y Soriano, 1980, p. 88), curiosamente, todos los relacionados con la polémica. Se tratan de además del propio Oliart, José Lladó (Transportes), Martínez de Genique (Agricultura) y Jiménez de Parga (Trabajo). Al día siguiente hay nuevo gobierno: Abril Martorell releva a Fuentes Quintana, Jaime Lamo de Espinosa en Agricultura, Rafael Calvo Ortega en Trabajo, Rodríguez Sahagún en Industria y Sánchez Terán en Transportes. Abril se proclamaba hombre fuerte del Gobierno y Lamo de Espinosa era de su confianza. Calvo Ortega y Rodríguez Sahagún son muy cercanos a Suárez (el último es su concuñado), y Sánchez Terán dejaba la compleja maquinaria del partido para ocupar una cartera. Se trataba de un paso atrás en el equilibrio de familias, y al mismo tiempo, en palabras de Abella, hacer un Gobierno "a medida" de Suárez y Abril (Abella, 2005, p. 354). El gran poder que obtendría este último sería a corto plazo un foco de tensión en el Gobierno que pasará factura como se analiza más adelante.

En la redacción de la Constitución tampoco hubo unanimidad total entre las filas del partido. Para empezar, costó nombrar a los tres miembros de la ponencia. Al final se optó por un equilibrio entre un hombre de Martín Villa (Gabriel Cisneros, familia azul), otro de Lavilla (Miguel Herrero, democristiano) y otro de Calvo-Sotelo (Pérez-Llorca, liberal-independiente). Inicialmente en lugar de Pérez-Llorca iba a ser miembro José María Martín Oviedo -abulense y de confianza de Suárez, pero Calvo-Sotelo intercedió para poner a uno de los suyos. $Y$ en las negociaciones entre los siete padres, hubo en algunos momentos diferencias entre los tres representantes de UCD. La que quizás más fricción suscitó fue la introducción del término "nacionalidades" en el artículo 2 de la Constitución. Miguel Herrero defendió su uso, pero Pérez-Llorca no se mostró favorable a ello, aunque finalmente se introdujese tras un acuerdo entre UCD, PSOE y Miquel Roca. Pero las diferencias en cuanto a la política territorial no solo quedaban ahí. Si bien Miguel Herrero defendía el reconocimiento del hecho diferencial para Cataluña y País Vasco, otro sector de UCD encabezado por Clavero Arévalo ya defendía la igualdad territorial y la oposición a cualquier tipo de estatus asimétrico para una comunidad en comparación con las otras.

Sin embargo, a pesar de las ya crecientes diferencias y rivalidades entre los miembros más destacados del partido, UCD consiguió mantenerse cohesionada a la hora de apoyar la Constitución y la estrategia de Suárez. Aunque parecía enormemente difícil, Hopkin parece indicar que esto se dio debido a la prevalencia del Gobierno sobre el grupo parlamentario (Hopkin, 2000, p. 110). Suárez y UCD se anotarían el éxito de la aprobación de la Constitución, pero el equilibrio expuesto en la "teoría del triciclo" se había derrumbado. 


\section{EI I Congreso de UCD}

El 19 de octubre de 1978 tiene lugar la celebración del I Congreso de UCD, evento que tenía como objetivo reforzar a Suárez como líder del partido, así como la definición de un marco programático sólido capaz de satisfacer a todos los sectores de la formación. No obstante, este segundo propósito iba a ser enormemente peliagudo para un partido que como se observa ya tiene una fragmentación interna considerable además de las rencillas existentes entre "barones". Aunque Suárez y Abril ganan a liberales, socialdemócratas, y algunos tácitos de cara a la distribución de poder planteada, los democristianos se opondrán a lo que según Huneuus ellos consideraban como un sistema presidencialista en el que el Presidente (Suárez) y el Secretario General (Rafael Arias-Salgado) tenían demasiado poder (Huneuus, 1985, p. 117). De hecho, llegaron a presentar una enmienda para cambiar así la organización de UCD, propuesta que fue mayoritariamente rechazada y que había sido impulsada por Ortega Díaz-Ambrona, a la sazón subsecretario del Ministerio de Justicia. Pero además de la división interna entre familias, también se daba una división "intrafamiliar"; esto quiere decir que las propias familias tampoco se ponían de acuerdo consigo mismas. $Y$ los propios democristianos no presentaron una postura homogénea en el Congreso. Si bien Álvarez de Miranda encabezó a aquellos que se oponían a la carga de poder que iban a adquirir Suárez y Arias-Salgado, otros democristianos como Íñigo Cavero, Javier Rupérez, Marcelino Oreja o Landelino Lavilla no pusieron objeción ninguna.

Por otro lado y para más inri, se produjo una intervención oral que suscitó polémica y molestias al sector socialdemócrata. El alcalde de Madrid José Luis Álvarez atacó duramente a la izquierda, palabras que chocaron con un sector que en plena Transición era partidario de las más estrechas formas de colaboración con partidos como el PSOE o el PSP de Tierno-Galván. Tampoco fueron bien vistas algunas decisiones tomadas por Arias-Salgado como Secretario General, que llegó a relevar de su cargo a Álvaro AlonsoCastrillo como responsable de las finanzas del partido, o a Guillermo Medina como Secretario de Información por diferencias con ellos. Arias-Salgado, procedente de la familia socialdemócrata, sería partir de entonces considerado como un "suarista".

No obstante, el resultado del Congreso presenta un balance muy positivo para Suárez sin perjuicio de la evidencia de la falta de cohesión interna que también se manifestó en la importante cita. El Presidente, arropado por personalidades del centro-derecha europeo como Margaret Thatcher, se proclama a efectos de Alonso-Castrillo como "el gran vencedor" (Alonso-Castrillo, 1996, p. 273).

\section{UCD ante las elecciones generales de 1979: guerra de listas}

Una vez aprobada la Constitución, se convocaron elecciones generales para marzo de 1979. La elaboración de las listas del partido supondrá un fuerte enfrentamiento interno en la formación. Se produce un auténtico choque entre partidarios de Martín Villa (azules) y los de Abril Martorell (abriles). La tensión fue aún mayor cuando el hermano del segundo, Joaquín Abril Martorell, fue nombrado por Suárez como director de campaña, algo que fue percibido por muchos como nepotismo. Por otro lado, el alcalde de Madrid José Luis Álvarez se empeñó en ser candidato al Congreso, aspiración que le 
negaron Fernando Abril Martorell y Otero Novas. Otro madrileño, el Presidente de UCD en Madrid Luis Ortiz, dimite cuando se entera que figura como número 11 en la lista de su propia provincia. Asturias también fue foco de tensión: Luis Vega Escandón se negó en rotundo en dejar el puesto de cabeza de lista a Calvo Ortega, entonces Ministro de Trabajo (Abella, 2005, p. 373).

En los comicios celebrados el 1 de marzo la UCD consigue 168 escaños, un total del $35 \%$ del voto (6.268.593 apoyos). Ganan tres diputados, los mismos que el PSOE (30\%) que se afianza como alternativa de gobierno con 121 diputados. Suárez necesita ocho parlamentarios más para ser investido. Le apoyan la Coalición Democrática de Fraga (10), el Partido Socialista Andaluz de Alejandro Rojas-Marcos (5) y el Partido Aragonés Regionalista (Hipólito Gómez de las Roces). Además, Convergència i Unió, en manos de Jordi Pujol, se abstiene. No obstante, la sesión en la que fue proclamado Presidente no estuvo exenta de polémica. No se produjo debate de investidura, lo que levantó gran revuelo y múltiples protestas hacia Suárez y Lavilla -ahora nuevo Presidente del Congreso-.

\section{| El principio del fin: todos contra Suárez y Suárez contra todos (1979-198I) |}

\section{Nuevo gobierno y fracaso en las municipales}

Tras renovar en el cargo, Suárez debe nombrar un nuevo Gobierno. Necesita hacer ajustes después de todo lo sucedido en los últimos meses, y decide formar un nuevo ejecutivo manteniendo en la medida de lo posible el equilibrio entre familias, pero deshaciéndose de los grandes barones de las mismas. De este modo, dejan el gobierno personalidades tan influyentes como Rodolfo Martín Villa, Pío Cabanillas y Fernández Ordóñez. La salida de Fernández Ordóñez fue considerada por algunos como un tanto a favor de los democristianos. El motivo de su marcha fueron las diferencias que había mantenido con el poderoso Abril Martorell. Fernández Ordóñez quiso presentar el año anterior el Plan Económico del Gobierno antes que los Presupuestos, iniciativa que chocó frontalmente con el Vicepresidente y Ministro de Economía. Ante una disyuntiva a la hora de elegir entre ambos, Suárez protegió a Abril y se deshizo (de momento) de Fernández Ordóñez en el nuevo gobierno (Jaúregui y Soriano, 1980, p. 100). Garrigues Walker perdía poder, pero seguía en el Ejecutivo como Ministro Adjunto al Presidente.

Tan sólo un mes después de las generales se celebrarían las primeras elecciones municipales de la democracia en España. El poder territorial comenzaba a adquirir un peso determinante en la política española, y UCD necesitaba consolidarse también en él. Sin embargo, los resultados de estas elecciones pueden ser considerados como el comienzo de un sinfín de descalabros por parte del partido de Suárez. La derrota más clamorosa fue la de la plaza de Madrid, donde José Luis Álvarez no consiguió revalidar ante el pacto entre Tierno-Galván y el PCE. Además, el PSOE también se hace con Barcelona (Narcís Serra), e importantes ciudades como Valencia, Alicante, Zaragoza, Valladolid o Vigo. EI PNV de Xabier Arzallus y Carlos Garaikoetxea consigue las tres 
grandes ciudades vascas (Bilbao, Vitoria y San Sebastián). Por su parte, el PCE obtenía tan solo una gran alcaldía pero que sería muy significante: Julio Anguita se convertía en regidor de Córdoba. Sevilla queda en manos de los andalucistas del PSA, mientras que la mayoría del resto de las grandes ciudades del sur son conquistadas por los socialistas. En Cataluña, la tónica general fue la victoria de los nacionalistas.

UCD conseguía al menos alcaldías como la de Oviedo, Santander, Logroño o Santiago, e incluso a nivel general habían obtenido 29.000 concejales frente a los 12.000 socialistas. Pero eso no quitaba la derrota simbólica ante el PSOE, que se convertía en el partido con mayor poder municipal en España, y que encima gobernaba en las dos grandes ciudades del país. Alonso-Castrillo cree que las causas de este fracaso se deben a los errores cometidos por Suárez entre las generales y municipales (como el no aceptar someter su investidura a debate) así como la publicación de las declaraciones fiscales de los más ricos por parte de Fernández Ordóñez, lo que según la autora habría reducido el apoyo de sectores de la derecha a UCD (Alonso-Castrillo, 1996, p. 359).

\section{División y errores en política territorial}

A lo largo de 1979 comienzan a redactarse y remitirse los Estatutos de Autonomía de País Vasco y Cataluña al Congreso. En el caso catalán, con la determinante presencia de Josep Tarradellas, al que se había nombrado President hasta la celebración de elecciones autonómicas en el proceso, la negociación se llevó a cabo entre representantes de UCD y de partidos catalanes. En cuanto al País Vasco, el Estatuto sale adelante gracias a la negociación entre Suárez y Carlos Garaikoetxea, presidente del PNV. Pero la concesión de estos derechos a estos dos territorios va a empujar al resto de comunidades a demandar el mismo trato. Las más fuertes en querer equipararse a País Vasco y Cataluña serán Galicia -al igual que las anteriores con una lengua propia muy extendida- y Andalucía, que se convertirá en un hervidero de problemas para el Gobierno, y especialmente para Suárez. El proceso en Galicia pudo facilitarse gracias a la acción de Pío Cabanillas, que tras dejar el gobierno actúa para la aprobación del Estatuto con la intensa colaboración de UCD, que en Galicia era dominante.

Pero el caso de Andalucía se va a convertir en un total quebradero de cabeza para el gobierno. UCD sufre una crisis de discurso, estrategia, unidad y objetivos que se convertirá en un fracaso sin precedentes para el partido. A diferencia de Galicia, en Andalucía el partido dominante era el PSOE, región de Felipe González y Alfonso Guerra, dónde además el Presidente de la Preautonomía era el socialista Rafael Escuredo. Por otro lado pero con pretensiones muy parecidas, el Partido Socialista Andaluz de Rojas-Marcos también ejercía gran presión sobre Suárez, al que habían apoyado en su sesión de investidura. Y la situación fue más allá con el sector regionalista andaluz originario del PSLA que se integró en UCD liderado por el entonces Ministro de Cultura Clavero Arévalo, que también defendía la aspiración autonomista andaluza.

Por otro lado, la situación del partido en la provincia andaluza donde habían recibido más apoyos en las generales de 1979 -un 44\%- se complicó por diversas tensiones internas. En noviembre de 1979, los senadores almerienses de UCD Torres Rollón y 
Ponce dimiten de sus cargos. La marcha de estos también vino acompañada de la de Pérez Company, Secretario General del partido en dicha provincia. Para QuirosaCheyrouze esta crisis provincial era otro caso más de cómo se dirimía el modelo de partido (Quirosa-Cheyrouze, 2002, p. 885).

En enero de 1980 la UCD trató de racionalizar el proceso autonómico. El partido quería poner freno a la vía rápida y dar preferencia a la vía lenta para el resto de autonomías tras Cataluña, País Vasco y Galicia. Ya que estaba previsto la celebración del referéndum andaluz para el 28 de febrero, la dirección tomó la decisión de hacer campaña por la abstención en la votación del Estatuto. El Gobierno no quiso que saliese adelante para evitar una cascada de configuración de gobiernos autonómicos: Andalucía era la primera de una larga cola.

Manuel Clavero Arévalo presentó su renuncia como Ministro tras el giro de guión de UCD y las trabas que ahora se establecían al autogobierno andaluz. A diferencia del partido a nivel nacional, la UCD de Sevilla así como de otros muchos lugares de Andalucía renunció a participar en la campaña. Según Abella, Suárez -siguiendo los consejos de Pérez-Llorca, Lavilla y Arias-Salgado- había cometido un grave error: la sociedad veía ilógico que el impulsor de la restauración hacia la democracia pidiera ahora al pueblo andaluz que no votase (Abella, 2005, p. 401). Además, el Gobierno había establecido como requisito para la aprobación la mayoría absoluta de los votos favorables en las ocho provincias, cosa que no ocurrió en Almería y Jaén, que se quedaron a las puertas. Aunque la votación no salía por esas dos provincias, la UCD debió rectificar y dar luz verde al Estatuto. Lo sucedido comenzó a ser conocido como el "error andaluz". Clavero hizo campaña por el Sí junto a Escuredo, quien llegó a ponerse en huelga de hambre para defender el voto favorable y denunciar la posición del Gobierno.

Y la situación se iba a complicar más aún con las cercanas citas electorales que tendrían lugar en marzo: el 9 se celebraban las primeras autonómicas vascas y el 20 las catalanas. Los resultados son muy negativos: en ambas comunidades se pierden un $8,5 \%$ de los apoyos en comparación con las generales de 1979. El PNV y $\mathrm{CiU}$ se hacían fuertes llevándose gran parte de los votos de UCD, que quedaba en una situación débil en regiones tan importantes como País Vasco y Cataluña, además de Andalucía.

Hacia la moción de censura: meses caóticos para UCD

Poco después de los batacazos de Andalucía, País Vasco y Cataluña, comienzan a generalizarse las críticas contra el gobierno de Suárez, sobre todo contra su equipo económico. Los ministros Carlos Bustelo (Industria) y Juan Antonio García Díez (Comercio), llegan a manifestar a Suárez sus profundas diferencias con Abril Martorell. Según Huneuus, esta vez Suárez no defiende a su Vicepresidente y baraja buscarle un sustituto que no llega a encontrar (Huneuus, 1985, p. 276).

Al mismo tiempo, los barones Martín Villa, Fernández Ordóñez y Garrigues Walker trasladan a Suárez su intención de ingresar de nuevo en el gobierno volviendo al 
equilibrio entre familias. De hecho, quieren vetar que Pérez-Llorca sea nombrado como un hipotético Vicepresidente $3^{\circ}$ a cargo de los asuntos regionales. De nuevo, Moncloa vivía una crisis de gobierno que no se resolverá hasta una nueva remodelación ministerial el 30 de abril. Abril Martorell continúa y consigue que los socialdemócratas Bustelo y García Díez que se habían enfrentado a él caigan del ejecutivo. Le suceden respectivamente Ignacio Bayón y Luis Gámir, abrilistas. Pérez-Llorca ocupaba la cartera de Administración Territorial pero sin Vicepresidencia ninguna.

Por otro lado, en estos meses se tratarán de aprobar el Estatuto de Centros Escolares y la Ley de Autonomía Universitaria (LAU), proyectos que van a provocar profundas divisiones en el partido. Las diferencias entre democristianos -que tenían a Otero Novas como Ministro de Educación- y los socialdemócratas -con Luis González Seara como Ministro de Universidades- van a fragmentar profundamente a la UCD. Si bien inicialmente el partido quería llegar a un consenso con el PSOE para sacar adelante el Estatuto de Centros Docentes, los democristianos y Otero Novas basculan hacia posiciones más conservadoras guardando el principio de libertad de enseñanza que según Hopkin implicaba en la práctica subvenciones para los centros de la Iglesia (Hopkin, 2000, p. 177), posición que apoyarían AP y $\mathrm{CiU}$. En el debate en el Congreso de la propuesta, Miguel Herrero -democristiano- defendió duramente el proyecto frente a socialistas y comunistas, discursos que según Abella levantaron recelo entre los socialdemócratas (Abella, 2005, p. 405). El Vicepresidente Abril Martorell se vio obligado a intervenir en el debate para garantizar a estos que la educación pública iba a gozar de una mejor posición con respecto a la privada.

El mismo término del principio de libertad de enseñanza iba a provocar la oposición esta vez por parte de los democristianos- a la Ley de Autonomía Universitaria que preparaba González Seara. En este documento se aceptaba el principio de enseñanza como servicio público, definición que sería considerada por los democristianos como una ofensa a lo privado y que les impulsó a ponerle trabas en su tramitación en el Congreso. Además, el hecho de que este texto legal hubiese comenzado a ser redactado por Íñigo Cavero (democristiano) cuando este era Ministro de Educación, y que por tanto iba a congeniar con las ideas de este sector, levantó aún más recelo entre los mismos. Para González Seara, el texto de Cavero era demasiado favorable a las universidades privadas (Powell, 2001, p. 285).

De otra parte, algunos autores coinciden en señalar que a partir de 1980 algunos poderes fácticos comienzan a plantearse la conveniencia de la continuidad de Suárez y UCD en el poder. Durante la I Legislatura la sociedad civil se hace más presente en la opinión pública, y con ello comienzan a ejercer una presión que se convertirá en un elemento más del sistema político español. La Iglesia Católica se pondrá del lado de los más conservadores del partido a raíz de las nuevas políticas en educación y divorcio. El principal promotor de este vínculo derecha-Iglesia será monseñor Antonio Innocenti, el nuncio apostólico de Juan Pablo II en Madrid. Por su parte, la patronal también se pone de lado de la derecha tras las reformas fiscales promovidas por Fernández Ordóñez que impulsaban la redistribución de la riqueza. Hopkin explica que los grandes sectores 
empresariales y la banca tratan de influir al Gobierno, para que, ahora que la democracia parecía consolidada, se derogasen algunas concesiones que se habían hecho a la izquierda (Hopkin, 2000). Pero Suárez no sigue este camino, y un partido de gobierno que no gozaba del apoyo ni de los sindicatos ni de la patronal iba a tener muy difícil su estabilidad en la Moncloa.

\section{Comienza el acoso y derribo: la moción de censura}

El 20 de mayo de 1980 Suárez se presentó en el Congreso de los Diputados para exponer el nuevo programa del gobierno que acababa de nombrar dos semanas antes. En esta sesión, Suárez prometió que todos los Estatutos estarían listos para 1983 y anunció nuevas medidas económicas. Según Prego, en realidad el Presidente trató de enmendar el error de no haber sometido su investidura a debate el año anterior (Prego, 2000, p. 98). Al día siguiente, Felipe González subió a la tribuna y anunció públicamente que el PSOE presentaba una moción de censura contra el Presidente Suárez.

Aunque el PSOE estaba lejos de la mayoría parlamentaria necesaria para derrocar al Presidente, Huneuus considera que los socialistas trataban en realidad de presentarse a sí mismos como una alternativa de poder además de recuperar el liderazgo interno del partido de González después de la celebración del XXVIII Congreso del PSOE (Huneuus, 1985, p. 287). Powell y Bonnin señalan que el PSOE aspiraba a convencer a los sectores más progresistas de UCD para llegar a la mayoría junto con la izquierda y algunos grupos nacionalistas (Powell y Bonnín, 2004, p. 173).

Tras los ataques en la tribuna de Felipe González y Alfonso Guerra, Suárez, que tenía la oportunidad de defenderse en una votación que prácticamente se sabía perdida, apenas hizo uso de los turnos de palabra a los que podía haber recurrido. Tan sólo tomó la palabra en tres ocasiones durante tan largo debate. Pero estas intervenciones tan solo fueron realizadas, las dos primeras para aclarar un hipotético pacto de gobierno UCDPCE al que había hecho mención Santiago Carrillo, y la otra como cierre final de la sesión en la que el Presidente hizo hincapié en la necesidad de un gobierno fuerte y reconoció que se habían cometido errores. Emilio Attard señala que desde la bancada del partido se alentó a Suárez para que pronunciase un discurso duro y que "rematara la suerte" (Attard, 1983, p.150). Pero no lo hizo, por lo que ya se veía la imagen de un Presidente derrotado moralmente.

El resultado de la votación fue el fracaso de la moción. Votaron a favor 152 diputados, en contra 166, y 21 se abstuvieron. 11 parlamentarios se ausentaron. De los 168 diputados de UCD hubo dos que rompieron la disciplina de partido y se pasaron a la abstención. Fueron Clavero Arévalo, alejado del partido desde el "error andaluz", y Joaquim Molins, que poco más tarde se uniría a CiU.

El fracaso político -que no parlamentario- de UCD en la moción de censura da comienzo a un período de decadencia marcado por las dimisiones y abandonos, los fuertes enfrentamientos internos y por la inestabilidad ministerial que ya se había convertido en algo habitual. 


\section{El principio del fin: la caída de Suárez}

A principios de julio de 1980, cinco semanas después de la moción de censura, tienen lugar una serie de reuniones en una finca propiedad del Canal de Isabel II de Madrid en la que Suárez convoca a la Comisión Permanente del partido. Esta había sido creada meses antes para dar voz a los grandes barones de las familias ideológicas que se habían quedado fuera del gobierno en abril de 1979. En estas reuniones en la que se conoció como "la casa de la pradera", Prego señala que se discute seriamente si Suárez debe seguir al frente de la formación y del Gobierno, debate acentuado después de que el propio Suárez dejase la habitación donde se debatía tal propuesta para que los barones hablasen con total libertad (Prego, 2000, p. 103). Los más críticos con el Presidente fueron Martín Villa y Garrigues. A pesar de los reproches a su líder, Martín Villa apuesta por su continuidad y Garrigues por “colegiar” (Abella, 2005, p. 249) el Gobierno -esto significaba el regreso de los barones al Ejecutivo-.

Una vez finalizado el verano, en septiembre Suárez anunció su nuevo gobierno. La gran variación del equipo fue la ausencia de Abril Martorell: tras años de confianza en el que fue su mano derecha, el Presidente prescindió de él cuando se produjo el fin del entendimiento entre ambos. Le sucedió en el cargo Calvo-Sotelo. Martín Villa fue nombrado Ministro de Administración Territorial, Fernández Ordóñez ocupó la cartera de Justicia, y Cabanillas Adjunto al Presidente. También estaba previsto el regreso de Garrigues al ejecutivo, pero falleció el 28 de julio después de una larga enfermedad. El nuevo gabinete parecía regresar, o al menos imitar, de algún modo, al primer gobierno del partido en 1977. Y esto, según González Casanova, chocaba ahora con los acontecimientos que se habían producido desde entonces: Martín Villa, conocido por estar en contra de la aceleración del proceso autonómico, ocupó Administración Territorial, y Calvo-Sotelo la Vicepresidencia Económica, "miembro de la oligarquía financiera” de un gobierno que quería llamarse socialdemócrata (González Casanova, 1986, p. 353).

La marcha de Abril Martorell fue todo un cambio en el gabinete de Suárez. Algunos autores coinciden en señalar que el origen de su caída fue un compromiso del Presidente con los barones en la reunión en "la casa de la pradera". No obstante, aquella defenestración guardaba razones más allá de las disputas del hasta entonces Vicepresidente $2^{\circ}$ con las familias. Para Powell y Bonnin, Abril Martorell mantuvo diferencias con Suárez a la hora de intentar favorecer a empresarios e Iglesia, referentes para él de lo que era base del electorado del partido (Powell y Bonnin, 2004, p. 174). Por su parte, Jaúregui y Soriano inciden en que el fin de Abril se veía venir desde las diferencias en la nueva política de pactos para buscar un socio que apoyase al gobierno. Suárez apostó por virar hacia $\mathrm{CiU}$, pero Abril fue favorable a un pacto con el PSOE (Jaúregui y Soriano, 1980, p. 184). A efectos de Navarro, la autora señala que la causa real que estaba detrás del fin de la relación era la misma razón por la que Suárez rompió con Osorio: Suárez quería disputarse los votantes de izquierdas con el PSOE (Navarro, 1995, p. 103). Dejaba el gobierno el que hasta entonces había sido su alter ego, un Vicepresidente que para muchos había tenido demasiados poderes. 
Adolfo Suárez sabe que el reajuste ministerial no era suficiente para renovar la imagen tan deteriorada desde la moción de censura, por lo que decidió tramitar una cuestión de confianza en el Congreso. No obstante, esta no dio los resultados esperados para el grupo parlamentario en el Congreso: Suárez no remontaba y dio una imagen parecida a la de la moción de censura. De nuevo tomaron el protagonismo sus ministros -ahora lo hizo especialmente Martín Villa-, aunque el Presidente no terminó mal parado gracias a la victoria en la votación por mayoría absoluta. Prego habla de un "éxito baldío" (Prego, 2000, p. 103) y Alonso-Castrillo de "victoria frágil" (Alonso-Castrillo, 1996, p.104). Se había conseguido vencer con los votos de CiU y el PSA de Rojas-Marcos, pero el debate vuelve a evidenciar la debilidad de Suárez y UCD frente a un Felipe González que arremetió duramente contra el gobierno y al que los espectadores habían prestado más atención en televisión.

Con el comienzo del nuevo curso político, fue sabido que Antonio Jiménez Blanco, hasta ahora Presidente del Grupo Parlamentario de UCD en el Congreso, sería nombrado Presidente del Consejo de Estado. Quedaba vacante un puesto de suma importancia para el partido en el parlamento, por lo que se convocó un proceso para elegir un sustituto. Santiago Rodríguez Miranda -de la familia socialdemócrata- se presentó con el apoyo de Suárez y Arias-Salgado. Tuvo como rival a Miguel Herrero, destacado democristiano que había sido uno de los redactores de la Carta Magna, y que conseguirá vencer con 103 votos frente a los 45 de Rodríguez Miranda.

Herrero se había convertido en el referente de todos los diputados de UCD descontentos con Suárez. Además de contar con el apoyo de Lavilla, Martín Villa, Cabanillas y Álvarez de Miranda, era el principal valedor de los "jóvenes turcos", un nutrido grupo de parlamentarios del partido que había surgido contra el poder de los barones pero que terminó convirtiéndose en un sector muy crítico con Suárez. Gascó Escudero va más allá y hace hincapié en que en realidad esta facción fue creada en torno a Abril Martorell para blindar a Suárez frente a los barones (Gascó Escudero, 2009, p. 84), por lo que el viraje de los "jóvenes turcos" habría sido aún mayor de confirmarse esta teoría. Esta nueva grave derrota para Suárez y Calvo Ortega -Secretario Generalevidenció el profundo descontento de los parlamentarios con su líder, sobre todo democristianos y liberales que se sentían desatendidos y abandonados (Hopkin, 2000, p. 192). Abella señaló que Suárez empezó a meditar desde este varapalo si dimitir o continuar en el cargo. El Presidente sabría de una operación que tendría como objetivo articular una gran centro-derecha conformado por democristianos, liberales, martinvillistas, y que a través de Miguel Herrero podría enlazar con los de Fraga (Abella, 2005, p. 445).

La desafección de los parlamentarios derivará en diciembre de 1980 a la publicación del Manifiesto de los 200, un documento firmado por destacados afiliados del partido que trataba, en palabras de Powell, de alejarse de posiciones izquierdistas para bascular hacia el conservadurismo (Powell, 2001, p. 287), y que iba a encaminado a ejercer gran influencia en el II Congreso de UCD que iba a tener lugar en febrero de 1981. Entre algunas de sus propuestas más destacadas, planteaban una mayor democratización del 
partido con una dirección colegiada (Contreras, 2016, p. 234). Ruiz Díez la denomina como una "operación crítica" que contaba con el apoyo de la CEOE y los bancos (Ruiz Díez, 2015, p. 14).

Pero poco antes de la celebración de esta importante cita para el futuro del partido, Adolfo Suárez anunció el 29 de enero de 1981 su dimisión como Presidente del Gobierno y de la UCD a través de RTVE.

Existen multitud de opiniones sobre que estaba detrás de la marcha de Suárez. Algunos refieren el ruido de sables de las FFAA, otros las presiones de los poderes fácticos como los empresarios o la Iglesia, el desgaste de su figura frente a una feroz oposición en manos de González y Guerra, o las diferencias con sus más incondicionales.

En opinión de Alonso-Castrillo, el nuevo contexto internacional de la década de los 1980 pudo haber influido en la caída de Suárez. Occidente oscila hacia el conservadurismo a partir de la victoria de Ronald Reagan en EEUU o la llegada de Wojtyla al Vaticano, presiones insalvables para un Presidente favorable a la solución negociada del conflicto Israel-Palestina (Alonso-Castrillo, 1996, p. 420). Por su parte, Navalón y Guerrero muestran una analogía entre Adolfo Suárez y Jimmy Carter en la que señalan que ambos llegan al poder como líderes progresistas en 1976, y se marchan -Carter en 1980 y Suárez en 1981- tras la incapacidad de hacer frente a la crisis económica y de contener el terrorismo -ETA en el caso español y la crisis de los rehenes en Teherán en el americano-(Navalón y Guerrero, 1987, p. 10).

La noche antes de su dimisión, Suárez convocó a la Comisión Permanente. En ella dejaba la puerta abierta a regresar para retomar el control de la formación y guiarla hacia una nueva victoria en las generales que debían celebrarse en 1983. Como se puede contemplar, efectivamente el "adiós" de Suárez era en realidad un "hasta luego", aunque, eso sí, no volvería a presentarse a la Presidencia bajo las siglas de UCD. Además, según Abella, poco antes de su dimisión habían comenzado a recogerse firmas para una nueva moción de censura, esta vez con el apoyo de diputados de UCD que también signaban el documento (Abella, 2005, p. 274). Luis Herrero narra que tiempo después el propio Suárez le desveló que él mismo se había enterado de dicha maniobra de nuevo impulsada por el PSOE, y que ahora iría a contar con apoyos de socialdemócratas, liberales o incluso de democristianos con el beneplácito de Miguel Herrero (Herrero, 2007, p. 213).

\section{EI II Congreso de UCD}

Como ya se ha señalado, la renuncia marcó por completo la celebración del II Congreso de UCD en Palma de Mallorca. El encuentro constató la división del partido en dos bloques; por un lado, un sector oficialista próximo a los más afines a Suárez con carácter continuista y centrista, y por el otro, un grupo crítico encabezado por algunos barones díscolos de tendencia democristiana. Los primeros reunieron a suaristas como AriasSalgado, Rodríguez Sahagún o Calvo Ortega; a los socialdemócratas liderados por Fernández Ordóñez; y a parte de los democristianos -como Íñigo Cavero o Javier Rupérez-; o incluso a barones como Pío Cabanillas o Martín Villa. 
Por otro lado, entre los críticos se encontraban Landelino Lavilla, Emilio Attard, Ignacio Camuñas, Gabriel Cisneros, Soledad Becerril, Marcelino Oreja o Jaime Mayor Oreja entre otros. Presentaron a Attard para presidir el II Congreso, votación que perdieron frente a Pérez-Llorca, que ya lo había hecho en el I Congreso celebrado en 1977. Tampoco vencieron con la candidatura de Lavilla a la Presidencia del partido, donde fue derrotado ante Rodríguez Sahagún. Los críticos defendían que UCD se asentase como formación que siguiera un estilo a lo holding company, término que según Hopkin encubría una propuesta para que el partido se asentase en el centro-derecha. Esta idea fue rechazada frontalmente por la dirección de la formación. Ni creían que hubiese espacio electoral para ello -dejando además el centro libre al PSOE-, ni mantendría la continuidad de socialdemócratas e independientes, ni habría autonomía en la toma de decisiones con respecto a los lobbies como la CEOE, la Iglesia y la CEE, o las asociaciones de padres de alumnos de las escuelas católicas, entre otros (Hopkin, 2000, 216).

No obstante, se constató que el sector crítico no era un grupo residual dentro del partido. Conformaba un clan que recibió más de un tercio de los apoyos en las dos votaciones mencionadas, y que estuvo representado en el Comité Ejecutivo con 7 de los 37 miembros. Suárez siguió en el mismo y recibió el cargo de Presidente de Honor del partido. Rodríguez Sahagún era el nuevo líder del partido y Calvo Ortega prosiguió como Secretario General. Pero en cambio, si bien hasta ahora el liderazgo del partido se correspondía asimismo con el candidato a la Presidencia del Gobierno, en este Congreso a propuesta de Suárez, se decide separar ambos cargos a fin de acabar con el sistema presidencialista que tantas quejas había recibido desde dentro. De este modo, Calvo-Sotelo fue ratificado como el sustituto de Suárez en la Moncloa. En opinión de Prego, Calvo-Sotelo era uno de los pocos miembros de la cúpula del partido que nunca se había sumado a algún movimiento crítico contra Suárez y que además se había mantenido alejado en buena medida de las disputas internas del partido, en parte gracias a su cargo como Ministro para las Relaciones con las Comunidades Europeas que le había mantenido muchas horas fuera de la vida política en Madrid (Prego, 2000, p. 119). Del mismo modo, tras la marcha de Abril Martorell, Calvo-Sotelo era Vicepresidente $2^{\circ}$ y sucesor natural de Suárez en el Ejecutivo, ya que no se contemplaba la posibilidad de que ascendiese dicho escalón el Vicepresidente $1^{\circ}$ Gutiérrez Mellado, totalmente ajeno al partido y con una actividad estrictamente orientada hacia la defensa y la seguridad nacional desde el primer gobierno de Suárez en 1976. Sin embargo, Huneuus aduce la elección de Calvo-Sotelo a la reunión de la Comisión Permanente mantenida la noche del 28 de enero antes mencionada. En ella, el autor señala que se produjo una votación en la que Calvo-Sotelo obtuvo los votos de Cabanillas, Arias-Salgado, Fernández Ordóñez, Pérez-Llorca, Martín Villa y Lavilla para suceder a Suárez en la Presidencia del Gobierno. Rodríguez Sahagún fue votado por Calvo Ortega y Abril Martorell, mientras que Landelino Lavilla fue votado por un Calvo-Sotelo que rehusó votarse a sí mismo (Huneuus, 1985, p. 332). Por su parte, Yárnoz señala que en esa misma madrugada Suárez pidió a Herrero y Alzaga que le apoyasen, pero terminaron ausentándose de la votación (Yárnoz, 1996, p. 552). 
Lo sucedido en Palma de Mallorca solo terminó por evidenciar la grave situación en la que se encontraba UCD. Tras la dimisión de Suárez y la escenificación de infinitas batallas internas, el evento que debía reunir al partido y plantear una nueva estrategia para mantenerse en el poder en las siguientes elecciones generales no dio resultado e incluso fue contraproducente en algunos sentidos. Lavilla había conseguido un $39 \%$ de los apoyos, lo que reflejaba la existencia de dos partidos en uno. Los democristianos no conseguían el giro a la derecha, pero provocaron un debilitamiento de los socialdemócratas, los menos votados en las listas al Comité Ejecutivo. Y por último, el sistema bicéfalo por el que coexistían un Presidente del partido y un Presidente del Gobierno no hizo sino agravar el liderazgo de la formación (Powell, 2001, p. 291).

\section{| Agonía y desaparición del partido (198|-1983)|}

\section{La investidura de Calvo-Sotelo}

Fue en plena sesión de investidura de Calvo-Sotelo tras la designación de este como candidato a la Presidencia por parte del Rey Juan Carlos cuando se produjo el golpe de estado fallido del 23-F. Se trataba de la segunda votación de la misma, ya que en la primera celebrada tres días antes no se había conseguido la mayoría absoluta necesaria.

Al mismo tiempo, ya en sus primeros días como candidato, Calvo-Sotelo recibe oposición por parte de algunos miembros de su partido. Según Abella, que estudia los boletines de Europa Press de aquellos días, al sector suarista -que seguía ostentando la inmensa mayoría del poder orgánico del partido- le molestó que el candidato no hubiese halagado lo suficiente la figura de Suárez durante sus intervenciones en su investidura (Abella, 2005, p. 550). Finalmente, en la repetición de la votación que se producirá después del golpe de estado, Calvo-Sotelo es elegido Presidente con mayoría absoluta. Además de los apoyos que ya tenía en la votación anterior, ahora todos los que se habían abstenido previamente votaban Sí para reforzar al ejecutivo en los primeros días de gobierno tras el golpe de estado. Comenzaba así un mandato tan breve como intenso.

\section{La Ley del Divorcio}

Calvo-Sotelo formó un nuevo gobierno mucho más reducido que el último de Suárez: eliminó siete ministerios que integró en otras carteras con el objetivo de hacer al ejecutivo más eficaz. De nuevo, se intentaba respetar el equilibrio entre las familias del partido: Martín Villa continuaba al frente de Administración Territorial, Fernández Ordóñez en Justicia, y Cabanillas sustituía a Arias-Salgado en Presidencia. Uno de los cambios más destacables del nuevo equipo era la eliminación de la Vicepresidencia Económica, puesto que había desempeñado el propio Presidente hasta antes de su nombramiento. El nuevo ministro de Economía era García Díez, socialdemócrata cuyo nombramiento generaría animadversión en la CEOE. Además, también se suprimía la Vicepresidencia para los Asuntos de la Defensa, por lo que por ahora no había ningún "segundo de a bordo" en Moncloa.

El proyecto legislativo que más división generó entre las filas de UCD en toda su breve historia fue la nueva Ley del Divorcio que trató de aprobar Fernández Ordóñez. Su 
antecesor en el cargo, Íñigo Cavero, ya intentó sacar adelante un proyecto que según Huneuus contaba con el apoyo de la Conferencia Episcopal Española, ya que no recogía el divorcio consensual (Huneuus, 1985, p. 342) al que se oponía la Iglesia. Sin embargo, cuando Fernández Ordóñez rescató el proyecto e incluyó ahora en el mismo esta modalidad de romper el matrimonio, la CEE muestra inmediatamente su oposición a la nueva propuesta de Ley. De este modo, los democristianos también se opusieron a dicha medida -aunque no ocurrió lo mismo con los liberales, más proclives al laicismo-. Tal fue así que, liderados por Miguel Herrero, lograron eliminar del documento el divorcio consensual en el Senado a través de lo que se conoció como "cláusula de dureza". Cuando la Ley retornó al Congreso ya en la última fase de su tramitación parlamentaria, Fernández Ordóñez volvió a hacer todo lo posible para rescatar el divorcio de común acuerdo. El ministro logró una votación secreta ante la enmienda que había presentado la izquierda para derogar aquella cláusula. Si bien el grupo parlamentario de UCD había ordenado a todos sus diputados votar en contra, una treintena de ellos rompió la disciplina de partido y votaron en el mismo sentido que el PSOE y el PCE. El divorcio consensual fue aprobado y Fernández Ordóñez lograba una relevante victoria parlamentaria a un proyecto en el que se había dejado todo su capital político.

Las consecuencias de lo sucedido llevaron a que Fernández Ordóñez perdiese el apoyo de muchos diputados del partido cercanos a Alzaga y Herrero, quienes crearon la Plataforma Moderada (formada por 39 diputados y 31 senadores), y que incluso pudieron a partir de entonces boicotear cualquier iniciativa del ministro. Ante tales diferencias, Fernández Ordóñez presentó su dimisión en agosto, dos meses después de que se aprobase la Ley en el Congreso. San Martín de Olalla se pregunta cómo fue posible que Calvo-Sotelo no apartase del cargo a Fernández Ordóñez cuando su proyecto estaba dinamitando totalmente al partido. El propio autor cree que pudo deberse a que una decisión así podría haber producido un nuevo desequilibrio que acabase con la marcha de los socialdemócratas del partido, o que directamente el propio Presidente en realidad estuviese de acuerdo con el proyecto (Marín de Santa Olalla, 2001, p. 545). Por su parte, Hopkin señala que en realidad Calvo-Sotelo poco podía hacer al no tener ningún tipo de poder ni responsabilidad en el partido, lo que le dejaba sin legitimidad para intervenir (Hopkin, 2000, p. 257).

Victorias y derrotas de un gobierno contra las cuerdas

Además de la Ley del Divorcio, en los primeros meses del gobierno de Calvo-Sotelo también había otras iniciativas fundamentales que seguían construyendo a la España democrática. En el verano de 1981 el Presidente logró firmar con Felipe González el Pacto Autonómico que al año siguiente daría pie al inicio de la tramitación LOAPA (Ley Orgánica de Armonización del Proceso Autonómico), que contaría con el rechazo de los nacionalistas vascos y catalanes. Las dudas sobre si dicho texto cumplía con lo estipulado en la Constitución llevarían a que Arias-Salgado y González acordasen paralizar su aplicación hasta que el TC se pronunciase sobre los recursos que se habían presentado. De esta forma, lo que parecía un resurgir del consenso en un aspecto tan crucial como la política territorial, ahora quedaba paralizado a la espera del órgano que salvaguardaba la Carta Magna. 
También en el verano de 1981 Calvo-Sotelo logró firmar un pacto histórico con los principales sindicatos -UGT y CCOO- y con la CEOE, al que se bautizó como Acuerdo Nacional para el Empleo (ANE). Según Alonso-Castrillo, desde los Pactos de la Moncloa no se alcanzaba un consenso entre las tres partes para decisiones de gran calado (Alonso-Castrillo, 1996, p. 489). No obstante, este avance común terminará volviéndose en contra de UCD cuando se presenten los Presupuestos Generales de 1982 a finales del año anterior. La CEOE se oponía a las subvenciones a los sindicatos, que iban a recibir 800 millones de pesetas. Desde entonces, a partir de las primeras elecciones siguientes a esta quiebra entre el Gobierno y la patronal -las andaluzas de mayo de 1982, la CEOE dejó de mostrar su apoyo a la UCD, y lo hizo a favor de la AP de Fraga.

En octubre de 1981 fue presentado el proyecto de adhesión a la OTAN. El Presidente anunció en su sesión de investidura que era su intención iniciar los trámites necesarios para esta incorporación histórica que suponía un cambio trascendental en la política diplomática española. Durante todos estos meses, el ministro de Exteriores PérezLlorca, y Javier Rupérez -secretario de Relaciones Internacionales de UCD- impulsaron las reuniones y acuerdos necesarios para la consecución de tal efecto. EI PSOE y el PCE se opusieron bajo el lema "OTAN, de entrada No", y exigieron un referéndum antes de entrar en la organización. El 29 de octubre el Congreso aprobó por mayoría absoluta la incorporación a la OTAN. Con 186 votos a favor, Calvo-Sotelo y Pérez-Llorca no solo consiguieron el apoyo de UCD, sino también el de CiU, la CD de Fraga, el PNV y Unión del Pueblo Navarro. Para Prego, el debate sobre la entrada en la OTAN marcó el primer plano de la vida política durante los dos años de gobierno de Calvo-Sotelo (Prego, 2000, p. 155).

Pero, a pesar de esta determinante victoria parlamentaria, el último trimestre de 1981 fue sangrante dentro de las filas del partido. Uno de los principales aceleradores de esta nueva crisis -o continuación de las ya innumerables anteriores- fueron los resultados de las elecciones gallegas del 20 de octubre de 1981. UCD perdió 240.000 votos en comparación con los resultados de las generales del 79 en Galicia, donde ahora ganaba la Alianza Popular de Fraga. Entre los errores en esta campaña, Huneuus achaca por un lado la división y la incapacidad organizativa del partido, y por el otro, la no inclusión de Gerardo Fernández Albor en las listas, (Huneuus, 1985, p. 374).

Dos semanas más tarde, Fernández Ordóñez anunció su marcha de UCD. Le siguieron varios socialdemócratas; once diputados y cinco senadores entre los que se encuentran González Seara y Javier Moscoso. No obstante, muchos de esta familia siguieron en la formación, entre ellos García Díez, Bustelo o Gámir. Fernández Ordóñez y los suyos constituyeron el PAD (Partido de Acción Democrática), que quería posicionarse en el espacio entre UCD y PSOE, pero que acabó presentándose a las generales de 1982 en las listas del PSOE.

Todos estos hechos conllevarán a que a finales de 1981 se produzca una remodelación tanto del partido como del gobierno. Tras la marcha de gran parte de los socialdemócratas y las diferencias con el sector suarista, Calvo-Sotelo se vio obligado a 
romper con la bicefalia instaurada en el Congreso de Palma -asumiendo así él mismo la Presidencia de UCD relevando a Rodríguez Sahagún-. Según Hopkin, este movimiento era inevitable si no quería que se produjese la desbandada de los democristianos de la Plataforma Moderada. Tal fue así que también optó por sustituir a Calvo Ortega en la Secretaría General del partido, puesto para el que propuso al democristiano Íñigo Cavero ante el Consejo Político. Los suaristas habían perdido su influencia dominante en el partido (Hopkin, 2000, p. 261).

Asimismo, el comienzo de 1982 estuvo marcado por un nuevo fracaso de otra iniciativa del gobierno. La Ley de Autonomía Universitaria (LAU), proyecto que venía siendo ya problemático dentro de las filas del partido cuando González Seara era Ministro de Universidades, fue definitivamente apartada por Mayor Zaragoza en abril de 1982. Ni Ortega Díaz-Ambrona ni él mismo fueron capaces de sacar adelante un texto que contaba con la oposición tanto de los más conservadores del partido como de los partidos de izquierda.

En definitiva, aún a pesar de ciertos éxitos como la entrada en la OTAN; el primer año de gobierno de Calvo-Sotelo estuvo marcado por fracasos políticos como la ANE y la LAU; el pronunciamiento de las divisiones internas del partido que trae consigo el comienzo de una desbandada de importantes personalidades de la formación como Suárez; y la constatación de que UCD estaba abocada a la derrota frente a un PSOE en pleno ascenso.

\section{La gran debacle}

Desde los primeros meses de 1982 el PSOE exigía elecciones anticipadas. Ante la clara previsión de una derrota frente a González, Calvo-Sotelo resistió en Moncloa.

En julio se produjeron una serie de reuniones entre Calvo-Sotelo, Suárez y Lavilla que tuvieron como fin la adopción de medidas para evitar la descomposición del partido. Calvo-Sotelo renunció aquí a la Presidencia de UCD, por lo que de nuevo se retornó a la bicefalia. Quiso que le sustituyera Lavilla, pero Suárez se opuso en rotundo. Tal fue así que Lavilla trató de retirar su candidatura, pero el Consejo Político le eligió con 144 votos frente a 65 abstenciones del sector suarista y ningún voto en contra. Por su parte, Abella señala que en realidad la marcha de Suárez era prácticamente irrevocable ya que venía preparando una nueva formación desde hacía tiempo (Abella, 2005, p. 577).

La elección del democristiano como líder del partido provocó el fin de las relaciones de Suárez con UCD y la creación del CDS. A la nueva formación de Suárez se le unieron algunos de sus más fieles incondicionales como Rodríguez Sahagún o Calvo Ortega, este último empujado no solo por la marcha de Suárez, sino también por la de Rodríguez Sahagún (González Martínez y Lafuente del Cano, 2019, p. 167). Según Huneuus, comienza una operación de "goteo" por la que varios partidos comienzan a tratar de seducir a destacados miembros de UCD para que dejasen la formación y se pasaran a sus filas (Huneuus, 1985, p.382). Y es que no solo CDS suponía una escisión de UCD; también estaba el PAD de Fernández Ordóñez, el nuevo Partido Demócrata Popular 
(PDP) y el Partido Demócrata Liberal (PDL). El PDP fue fundado por Óscar Alzaga en el mismo mes que Lavilla era elegido Presidente de UCD. Se trataba de un importante sector de los democristianos, por lo que en sus primeros días el nuevo Presidente de UCD ya había perdido una notable parte de sus apoyos. EI PDP contaba con Otero Novas, Reguera o Carriles, tres exministros de UCD. Por otro lado, el PDL fue fundado por Antonio Garrigues Walker, hermano del difunto Joaquín Garrigues Walker. Esta formación aglutinó a tan solo unos pocos del sector liberal de UCD, pero también contribuía al ruido de la sangrante descomposición.

Calvo-Sotelo terminó claudicando en agosto. Era imposible aguantar hasta 1983 con la inmensa desbandada de diputados y senadores que se había producido en tan solo un mes: hacia el centro-izquierda (PAD), el centro-derecha (PDP y PDL), o el mismo centro (CDS), la UCD estaba prácticamente desmembrada. Había comenzado el éxodo de unas familias que nunca llegaron a congeniar cómodamente.

La labor de Lavilla como nuevo Presidente y candidato a la Moncloa fue intentar dejar a la UCD en una buena posición. Todos sabían que iban a perder frente al PSOE, pero al menos debían obtener un holgado grupo parlamentario que les permitiera realizar una importante labor de oposición. Poco antes de las elecciones se conoció que el PAD concurriría con el PSOE y el PDP con AP. Miguel Herrero se fue directamente a las filas de Fraga sin necesidad de pasar por las de Alzaga. Suárez fue en solitario con un partido al que tuvo que estructurar en tiempo récord. En definitiva, el centro político se dividía entre los que acudían al eje izquierda-derecha, y los que se mantenían fieles al espacio entre AP y el PSOE con unas expectativas muy bajas. Wert señala que algunos miembros como Martín Villa o Marcelino Oreja defendían concurrir junto con Fraga en una gran coalición de centro-derecha, propuesta que Lavilla rechazó para precisamente no abandonar el centro político (Wert, 1984, p.68).

Finalmente, la derrota fue todavía mayor de la esperada. Si bien la UCD obtuvo 168 diputados en 1979, ahora tan solo conseguía 11, perdiendo también todos sus senadores. Lavilla solo consiguió mantener 1.400 .000 votos de los 6.200 .000 con los que Suárez se valió en las anteriores elecciones. Este último sufrió un descalabro aún peor: solo conseguía su escaño por Madrid y otro por Ávila para Rodríguez Sahagún con 600.000 votos en toda España. Felipe González arrasó con 202 diputados y más de diez millones de votos. Fraga ocupaba gran parte del espacio de UCD y ascendía hasta los 107 diputados, lo que le convertía en el nuevo líder de la oposición.

En definitiva, Alonso-Castrillo señala que el descalabro de UCD es uno de los más inauditos en la historia de los partidos del mundo occidental (Alonso-Castrillo, 1996, p. 537), Hopkin lo considera catastrófico (Hopkin, 2000, p. 263), y Huneuus destaca que aquella derrota era el fin de UCD (Huneuus, 1985, p. 246).

\section{El fin de UCD}

En diciembre de 1982 se convocó un Congreso Extraordinario en Madrid para reorganizar a un partido que estaba acabado. Asistieron a él pocas personalidades 
influyentes a diferencia de las otras grandes citas de la formación; Calvo-Sotelo y Cabanillas optaron por no acudir a un evento que era prácticamente el prólogo de la defunción de UCD. Sí estaban allí dos sectores que se iban a enfrentar incluso en las horas más bajas de la formación. La familia de los azules, que se había quedado en su mayoría en el partido liderados por Martín Villa y Gabriel Cisneros, exigió responsabilidades a los democristianos de Lavilla y Cavero. Los últimos conservaron la dirección del partido -donde Cavero fue reemplazado en la Secretaría General por Ortega Díaz-Ambrona, pero el grupo parlamentario sería dirigido por Cisneros.

Con el comienzo de 1983, la dirección del partido vio prácticamente imposible presentarse a las municipales que iban a tener lugar en mayo. Ahogada por los bancos, a los que la coalición debía unos 5.000 millones de pesetas (Hopkin, 2000, p. 288), la cúpula decidió disolver la formación en febrero de 1983. Aunque los azules se oponían, la decisión de los democristianos desde los órganos de poder del partido puso fin a una formación que para Alonso-Castrillo tuvo un "profundo éxito como gobierno, pero un estrepitoso fracaso como partido” (Alonso-Castrillo, 1996, p. 544).

\section{| Conclusiones: una larga sombra de la división interna del centro que se perpetúa |}

Desde sus orígenes, UCD estaba dividida en sectores que venían de diferentes partidos políticos que se agruparon en torno a una coalición que sirvió como herramienta para ganar las elecciones de 1977. La existencia de estos grupos traería consigo la formación de numerosas familias políticas que trabajaban por sus intereses dentro del partido, y que trataban de que su ideología fuese la dominante en la formación. Liderados por sus propios barones, en UCD convergían democristianos, liberales, socialdemócratas, azules, suaristas, abrilistas, e incluso regionalistas. Este inasumible conglomerado de visiones sobre la política e intereses familiares no solo provocaría enormes tensiones en la distribución de poder en el gobierno (ministerios) y en el partido (cargos orgánicos), sino que terminaría afectando a grandes iniciativas legislativas que acabaron fracasando fruto de esta división interna. Son claro ejemplo de ello las diferencias en el Estatuto de Centros Escolares, la LAU, el ANE, y por supuesto, la fatídica Ley del Divorcio. Asimismo, a la mínima que el gobierno o el partido basculasen hacia la derecha o la izquierda, se suscitaban recelos en el sector contrario, lo que dificultó enormemente la toma de decisiones de gran calado en numerosas ocasiones. Desde su nacimiento, el partido padecía de una debilidad congénita.

Este estudio nos brinda, por tanto, el aporte de una paradoja que parece extenderse hasta nuestros días. La sombra de esta imposibilidad de afianzarse como partido se ha perpetuado en el resto de formaciones del mismo espectro político que le han seguido. Ahí están, los casos del Centro Democrático y Social (CDS), o de Unión, Progreso y Democracia (UPyD, proyectos que no lograron estabilizarse políticamente y que tuvieron un recorrido político casi efímero. $Y$ actualmente, el caso de Ciudadanos- 
Partido de la Ciudadanía, que en 2021 vive una gravísima crisis que pone en cuestión su continuidad con el suficiente apoyo electoral y representación parlamentaria.

UCD ha pasado a la historia por ser una de las formaciones políticas que hizo posible la consecución efectiva de la Transición. El cambio de un régimen dictatorial a uno democrático tuvo como uno de sus principales protagonistas a un partido tan trascendental como efímero. Su historia, sus líderes, sus estrategias y sus iniciativas marcaron y marcan la sociedad española. Este estudio ha tratado de demostrar cómo la división interna se convirtió, quizás, en la principal causa del estrepitoso fracaso y descalabro de un partido que, aunque por poco tiempo, lo fue todo pilotando el tránsito hacia nuestro actual régimen constitucional y Estado de Derecho.

\section{Referencias}

Abella, C. (2005). Adolfo Suárez. Editorial Folio.

Alonso-Castrillo, S. (1996). La apuesta del centro: historia de la UCD. Alianza.

Attard, E. (1983). Vida y muerte de UCD. Planeta.

Caciagli, M. (1989). La parábola de Unión de Centro Democrático. En Tezanos, J.F.; Cotarelo, R.; y De Blas, A. (eds.). La Transición Democrática Española (pp. 389-432). Sistema.

Contreras, E. (2016). Suárez. Acoso y derribo. La Esfera de los Libros.

Gascó Escudero, P. (2019). UCD-Valencia: Estrategias y grupos de poder políticos. Universidad de Valencia.

González Casanova, J.A. (1986) El cambio inacabable (1975-1985). Anthropos.

González Martínez, C., y Lafuente del Cano, J. (2019). Rafael Calvo Ortega y la crisis del centro político: la disolución de UCD desde una perspectiva suarista. En Navajas Zubeldia, C. (ed.). El reinado de Juan Carlos I (1975-2014): actas VI Congreso Internacional de Historia de Nuestro Tiempo (pp.161-170).

Herrero, L. (2007). Los que le llamábamos Adolfo. La Esfera de los Libros.

Hopkin, J. (2000). El partido de la Transición. Ascenso y caída de la UCD. Acento.

Huneuus, C. (1985). La Unión de Centro Democrático y la Transición a la Democracia en España. Centro de Investigaciones Sociológicas.

Jáuregui, F. y Soriano, M. (1980). La otra historia de UCD. Emiliano Escolar.

Linares, A.L. (2013). El grupo Tácito en la Transición a la Democracia. Aportes, 83, 6887. 
Martín de Santa Olalla, P. (2001). La ley del divorcio de 1981 en perspectiva histórica Espacio, Tiempo y Forma, 14, pp. 519-554.

Morán, G. (2009). Adolfo Suárez. Ambición y destino. Debate.

Navalón, A. y Guerrero, F (1987). Objetivo Adolfo Suárez. Espasa Calpe.

Navarro, J. (1995). Nosotros la Transición. Temas de Hoy.

Powell, C. y Bonnín, P. (2004). Adolfo Suárez. Cara y Cruz.

Powell, C. (2001). España en democracia, 1975-2000. Plaza y Janés

Prego, V. (2000). Presidentes. Veinticinco años de historia narrada por los cuatro jefes de Gobierno de la democracia. Plaza y Janés.

Quirosa-Cheyrouze, R. (2002). Un antecedente en la crisis de UCD: la renuncia de dos senadores. En Navajas Zubeldia, C. (ed.), Actas del IV Simposio de Historia Actual pp. 799828.

Wert, J.I. (1984). La campaña electoral de octubre de 1982: el camino del cambio. Reis: Revista española de investigaciones sociológicas, 28 (pp.63-84).

Yárnoz, C. (1996). El ocaso de UCD. En Prieto, J.; Juliá, S.; y Praderá, J. (eds). Memoria de la Transición (pp. 511-518).

\section{| Nota biográfica |}

José Ignacio Villar Romero es técnico de Apoyo y Gestión de I+D+i de la Universidad de Córdoba, adscrito a los grupos HUM808 Regulación Social e Instituciones en Andalucía; y SEJ372 Democracia, Pluralismo y Ciudadanía. Doble Graduado en Historia + Ciencia Política y Gestión Pública por la Universidad Rey Juan Carlos. Colaborador del proyecto de investigación: Instrumentalización de iniciativas sociales durante el primer franquismo en Andalucía (1939-1959): incidencia en las poblaciones afectadas en Córdoba, particularmente sobre los asistidos institucionalmente, niños y jóvenes en peligro, presos y locos. Ha trabajado anteriormente en consultoría pública, colaborando en la redacción de licitaciones y contratos. Ha sido asesor histórico para la Simulación de la Transición Española (SITE), organizada por la Asociación Estudiantil de Simulaciones Parlamentarias (ASESP). Obtuvo el premio a Mejor Parlamentario en Simulación de la Cámara de los Comunes Británica (SICCOB). 